\title{
Treatment results of incomplete chemoradiotherapy in locally advanced cervical cancer
}

This article was published in the following Dove Press journal:

OncoTargets and Therapy

29 March 2013

Number of times this article has been viewed

Ying Gao',*

Fei $\mathrm{GaO}^{2, *}$

Zi Liu'

Li-ping Song'

'Department of Radiotherapy Oncology, First Affiliated Hospital of Medical College of Xi'an Jiaotong University, Xi'an, People's Republic of China; ${ }^{2}$ Second Affiliated Hospital of Medical College of Xi'an Jiaotong University, Xi'an, People's Republic of China

*These authors contributed equally to this work
Correspondence: Zi Liu, Li-ping Song Department of Radiotherapy Oncology, First Affiliated Hospital of Medical College of Xi'an Jiaotong University, Xi'an 7I 006I, People's Republic of China $\mathrm{Tel}+8602985324019$

Email liuzmail@I63.com,xasIp@I26.com
Objective: Regimens that combine chemotherapy and radiotherapy increase toxicity and compromise a patient's ability to adhere to the treatment plan. We evaluated the efficacy and safety of a partially completed chemoradiation regimen prescribed for locally advanced carcinoma of the cervix.

Methods: Medical records of 156 patients with locally advanced cervical cancer stage IIB-IVA who received chemoradiation with cisplatin $\left(40 \mathrm{mg} / \mathrm{m}^{2}\right)$ and 5-fluorouracil $(500 \mathrm{mg} /$ $\mathrm{m}^{2}$ ) from October 2006 to October 2008 were collected. The treatment protocol called for two cycles of chemotherapy. External beam radiation therapy was administered using a $10-\mathrm{MeV}$ electron beam. Local control, disease free survival, overall survival, and toxicities were evaluated.

Results: With a median follow-up of 37.5 months, 89 patients (57\%) completed the planned protocol. Sixty seven patients (43\%) completed fewer than two cycles. The 3-year local control rate was significantly better in the patient group that completed the prescribed plan $(92.1 \%$ compared to $80.6 \% ; P=0.033$ ). No statistical significance was observed between the groups that completed or did not complete the two cycle protocol with regard to disease free survival (80.9\% and $73.2 \%$, respectively; $P=0.250$ ), overall survival $(84.3 \%$ and $79.1 \% ; P=0.405)$, and progression survival $(3.4 \%$ and $3.0 \% ; P=0.892)$. Differences in acute hematologic toxicity and subcutaneous toxicity were observed between the two groups.

Conclusions: Completion of two cycles of 5-fluorouracil and cisplatin with radiotherapy was effective, safe, and responsible for better local control; toxicity was acceptable. Disease free survival and overall survival were similar between the treatment groups.

Keywords: cervical cancer, chemoradiotherapy, 5-fluorouracil, cisplatin, local control, toxicity

\section{Introduction}

Cervical cancer is the fourth leading cause of cancer death among women worldwide. The main treatments consist of radiation therapy, surgery, chemotherapy, or a combination of these options. In advanced stages (IIB-IVA), radiation therapy concurrent with chemotherapy (ie, chemoradiation or chemoradiotherapy) is standard, and platinum-based chemotherapy protocols are preferable. According to the National Cancer Institute, the benefit of concurrent cisplatin-based chemoradiation in women with cervical cancer exceeds that of radiotherapy alone. ${ }^{1}$

However, in patients receiving adjuvant chemoradiotherapy, hematologic and gastrointestinal toxicities are risk factors for reduced adherence to the regimen, ${ }^{2}$ as some patients cannot tolerate all courses. ${ }^{3}$ It must be recognized that both the disease 
and the effects of treatment compromise in many ways the wellbeing and quality of life of these women. Although the most common regimens are a combination of 5-fluorouracil (5-FU) and cisplatin administered at three to 4-week intervals, the optimal dose and dosage have not been determined. ${ }^{3-6}$

We conducted the present retrospective study to evaluate the efficacy of incomplete treatment in chemoradiation with 5-FU and cisplatin for locally advanced cervical carcinoma. Our primary goal was to compare treatment outcomes, including survival and recurrence rates, in an effort to determine the superior chemoradiotherapy regimen. We also evaluated patients' ability to adhere to the planned regimen and associated toxicities.

\section{Materials and methods Patients}

The Ethics Committee of the First Affiliated Hospital of Medical College of Xi'an Jiaotong University approved this study. Data were collected retrospectively from the records of 156 consecutive patients with locally advanced cervical cancer who had been treated with combined radiotherapy and chemotherapy from October 2006 to October 2008. All patients had provided written informed consent for treatment.

The patients included in this study presented with International Federation of Gynecology and Obstetrics stages IIB-IIIB (IIB: cervical carcinoma invades beyond the uterus with obvious parametrial invasion, but not to the pelvic wall or to the lower third of the vagina; IIIB: tumor extends to the pelvic wall, involves the lower third of the vagina and/or causes hydronephrosis or non-functioning kidney), good performance status (Eastern Cooperative Oncology Group score of 0 [asymptomatic], or 1 [symptomatic but ambulatory]), no uncontrolled concomitant disease, no connective tissue disease, and no prior irradiation. For evaluation, the patients were assigned to either of two groups. Patients in group I completed two cycles of the chemotherapy regimen, and those in group II received fewer than two cycles.

\section{Radiation therapy}

External beam radiation therapy was delivered in a conventional fraction (1.8 Gy/fraction, 5 fractions/week) using a 10-MV photon beam from a linear accelerator. A total dose of 50.4 Gy was administered to the entire pelvis. This was followed by intracavitary brachytherapy at a dose of 5 Gy in 4 fractions to point A (the paracervical triangle in the medial edge of the broad ligament where the uterine vessels cross the ureter) which was delivered by a remote afterloading system. External beam radiation therapy was interrupted if the white blood cell count fell below $1000 / \mathrm{mm}^{3}$ or if platelets fell below $50,000 / \mathrm{mm}^{3}$, and was resumed once counts rose above these levels.

\section{Chemotherapy}

Cisplatin and 5-FU were the chemotherapy agents administered. Cisplatin was given in a dose $40 \mathrm{mg} \cdot \mathrm{m}^{-2} \cdot \mathrm{d}^{-1}$ for 3 days; 5 -FU was given at $500 \mathrm{mg} \cdot \mathrm{m}^{-2} \cdot \mathrm{d}^{-1}$ continuous infusion from the first to the fifth day with radiotherapy. A 3-week rest separated the two cycles.

Patients were seen weekly by a physician for a physical examination and a complete blood count test. Chemotherapy was stopped if creatinine clearance was $<30 \mathrm{~mL} / \mathrm{min}$, and interrupted if patients had $\geq$ grade 3 gastrointestinal toxicity, the total white blood cell count was $\leq 4000 / \mathrm{mm}^{3}$, or platelets were $\leq 100,000 / \mathrm{mm}^{3}$. Granulocyte colony-stimulating factors were used when absolute neutrophil count fell $\leq 500 / \mathrm{mm}^{3}$ or total white blood cell count fell $\leq 1000 / \mathrm{mm}^{3}$.

\section{Toxicity}

During treatment, toxicities were assessed weekly and graded in accordance with the National Cancer Institute Common Terminology Criteria of Adverse Events (version 3.0): 1, mild; 2 , moderate; 3 , severe; and 4, lifethreatening or disabling. ${ }^{7}$

\section{Statistical analyses}

Data were stored and analyzed using SPSS version 17.0 software (IBM Corporation, Armonk, NY, USA). The Kaplan-Meier approach and the log-rank test were used to compare survival profiles between the two patient groups. $P<0.05$ was considered significant for all statistical analyses.

\section{Results}

\section{Patient characteristics}

The median age of the patients in groups I and II at the time of diagnosis was 50 and 51 years, respectively. In total, 156 patients were included from October 2006 to October 2008 (Table 1). One hundred and one (64.7\%) patients were diagnosed as International Federation of Gynecology and Obstetrics stage IIB, and 55 (35.3\%) in stage IIIB. One hundred and sixteen patients had squamous cell carcinoma (74.4\%) and $37(23.7 \%)$ had adenocarcinoma. The histology of the remaining three patients $(1.9 \%)$ is unknown. 
Table I Clinicopathology of patients by treatment group

\begin{tabular}{llll}
\hline & Group I $^{\text {a }}$ & Group II $^{\mathbf{b}}$ & P-value \\
\hline $\begin{array}{l}\text { Age (mean year, range) } \\
\text { Duration of treatment } \\
\text { (median days, range) }\end{array}$ & $50,31-68$ & $5 \mathrm{I}, 33-69$ & 0.96 \\
FIGO stage (n, \%) & $49,42-62$ & $50,43-65$ & 0.89 \\
$\quad$ IIB & & & \\
$\quad$ IIIB & $63,70.1$ & $38,56.7$ & 0.069 \\
Histology (n, \%) & $26,29.9$ & $29,43.3$ & \\
$\quad$ Squamous cell carcinoma & $61,68.5$ & $55,82.1$ & 0.158 \\
$\quad$ Adenocarcinoma & $26,29.2$ & II, I6.4 & \\
$\quad$ Unknown & $2,2.3$ & I, I.5 & \\
\hline
\end{tabular}

Notes: ${ }^{T}$ Two cycles, $\mathrm{n}=89$; bewer than two cycles, $\mathrm{n}=67$.

Abbreviation: FIGO, International Federation of Gynecology and Obstetrics.

Eighty-nine patients (57\%, group I) completed the two cycles of cisplatin $\left(40 \mathrm{mg} / \mathrm{m}^{2}\right)$ and 5 -FU $\left(500 \mathrm{mg} / \mathrm{m}^{2}\right)$, while 67 patients ( $43 \%$, group II) received fewer than two cycles. The most common causes for not completing the chemotherapy plan were neutropenia $(95.6 \%)$, anemia plus neutropenia $(57.8 \%)$, and others $(1.3 \%)$.

\section{Survival and local control}

With a median follow-up time of 37.5 months (range 7-65 months), the local control for groups I (completed two cycles) and II (completed $<2$ cycles) were $92.1 \%$ and $80.6 \%$, respectively (Table 2 and Figure 1). Disease-free survival for the two groups was $80.9 \%$ and $73.2 \%$, overall survival was $84.3 \%$ and $79.1 \%$, and progression survival was $3.4 \%$ and $3.0 \%$. As shown by univariate analyses, only in local control was there a statistically significant difference between the two groups $(P=0.033)$.

Considering only stage IIB patients in the treatment groups, local control was achieved by $96.8 \%$ in group I and $86.8 \%$ in group II, while disease-free survival was $85.7 \%$ and $78.9 \%$, respectively, and overall survival rates were $88.9 \%$

Table 2 Three-year local control, disease free survival, and overall survival rates stratified by patient group

\begin{tabular}{llll}
\hline & $\begin{array}{l}\text { Group I } \\
\text { (n, \%) }\end{array}$ & $\begin{array}{l}\text { Group II }^{\mathbf{b}} \\
(\mathbf{n}, \%)\end{array}$ & P-value \\
\hline Local control rates & $82,92.1$ & $54,80.6$ & 0.033 \\
Stage IIB & $61,96.8$ & $33,86.8$ & 0.130 \\
Stage IIIB & $21,80.8$ & $21,72.4$ & 0.467 \\
Disease free survival rates & $72,80.9$ & $49,73.2$ & 0.250 \\
Stage IIB & $54,85.7$ & $30,78.9$ & $0.58 I$ \\
Stage IIIB & $18,69.2$ & $19,65.5$ & 0.769 \\
Overall survival & $75,84.3$ & $53,79.1$ & 0.405 \\
Stage IIB & $56,88.9$ & $32,84.2$ & 0.496 \\
Stage IIIB & $19,73.0$ & $21,72.4$ & 0.956 \\
Progression survival & $3,3.4$ & $2,3.0$ & 0.892 \\
\hline
\end{tabular}

Notes: ${ }^{\text {TTwo }}$ cycles, $n=89$; bewer than two cycles, $n=67$. and $84.2 \%$ (Table 2 and Figure 1). For stage IIIB patients, local control was achieved by $80.8 \%$ and $72.4 \%$ in groups I and II, respectively, while disease-free survival was $69.2 \%$ and $65.5 \%$, and overall survival rates were $73.0 \%$ and $72.4 \%$, respectively.

\section{Toxicities}

Grade 3-4 anemia and neutropenia was found in 11 and 20 patients, respectively (Table 3 ). In group I, three and six patients developed grade 3-4 anemia and neutropenia, respectively, while in group II, 8 and 14 patients developed these side effects, respectively. The differences in acute grade 3-4 hematologic toxicity between the two patient groups was significant ( $P=0.038$ for anemia; $P=0.009$ neutropenia). Fifteen patients developed grade 1-2 thrombocytopenia.

There was no statistical difference between the patient groups in terms of the incidence of grade 1-2 subcutaneous toxicity (Table 4). However, while none of the patients in group I developed grade 3-4 subcutaneous toxicity, in group II 8 of $67(11.9 \%)$ developed this condition $(P=0.001)$.

\section{Discussion}

Based on five large randomized trials in which $30 \%$ to $50 \%$ improvements in survival rates were shown, cisplatin-based chemoradiotherapy has become the standard treatment for women with locally advanced cervical cancer. ${ }^{8-12}$ In addition, for these women chemoradiotherapy with concurrent cisplatin and 5-FU had several survival advantages over extended field radiation alone or oral administration of hydroxyurea. ${ }^{9,12}$

The most popularly applied chemotherapeutic regimens are weekly cisplatin or combined cisplatin and 5-FU at three to 4-week intervals, but the optimal dose and dosage have not yet been definitively determined because of the toxicities associated with these agents. In GOG-120, GOG-165, and other similar clinical trials, only $50 \%$ to $70 \%$ of patients were able to complete six cycles of cisplatin as scheduled without dose decreases or interval changes. ${ }^{13,14}$ In addition, a study by Ryu et a ${ }^{15}$ found that triweekly cisplatin-based radiochemotherapy had more survival benefit and less associated neutropenia than a weekly treatment for locally advanced cervical cancer. Yet the association between completion of scheduled treatment and prognosis has not been investigated as thoroughly, although a series of literature reports have revealed a reduced adherence rate for chemoradiation with cisplatin. 
A

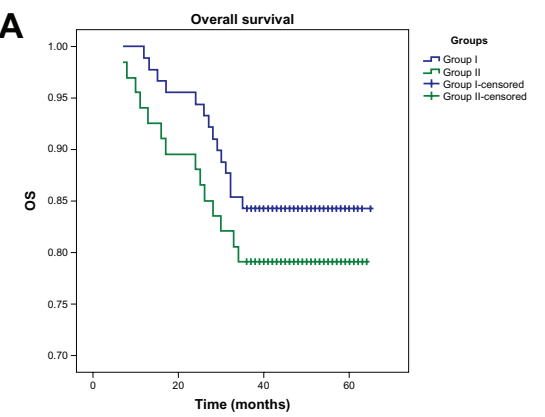

D

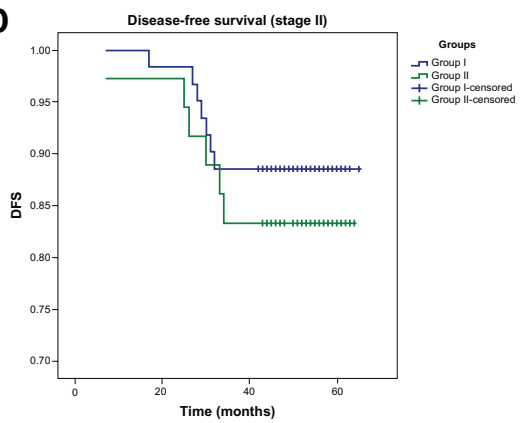

B
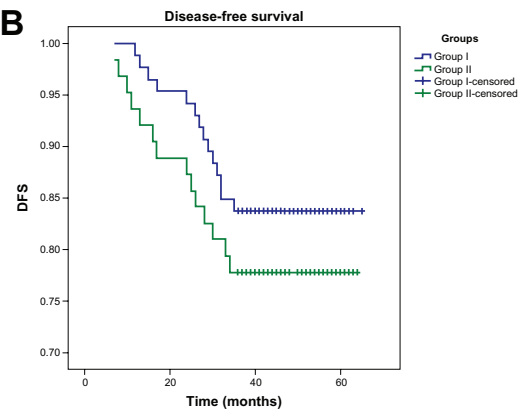

E
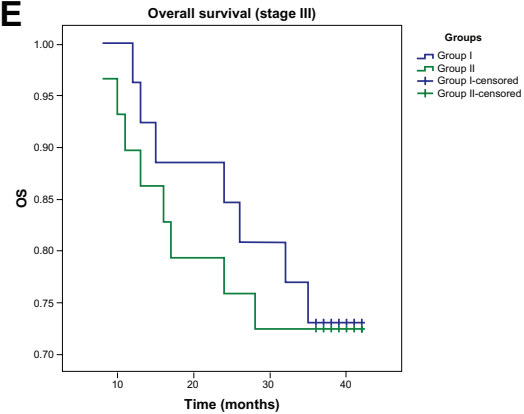

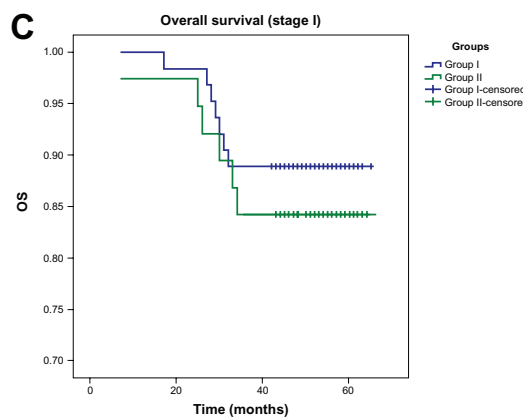

$\mathbf{F}$

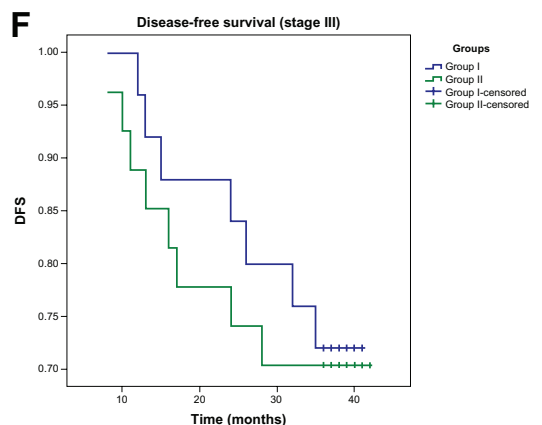

Figure I Survival analysis in locally advanced cervical carcinoma, stratified by groups I and II $(P>0.05$, all). (A) Overall survival curves. (B) Disease free survival curves. (C) Overall survival curves of stage IIB patients. (D) Disease-free survival curves of stage IIB patients. (E) Overall survival curves of stage IIIB patients. (F) Disease free survival curves of stage IIIB patients.

The Some trial found that chemotherapy with 5-FU and cisplatin when added to external beam and intracavitary radiation significantly improved survival in locally advanced cervical cancer. ${ }^{16}$ The addition of 5-FU was based on the fact that this agent and its metabolites inhibit angiogenesis induced by vascular endothelial growth factor, and thus has an antitumor effect on cervical and colon cancers. ${ }^{17,18}$ A statistically significant benefit was also shown for adjuvant pelvic radiation concurrent with 5-FU and cisplatin in the treatment of patients with high risk factors found at surgery. ${ }^{1}$ In addition, preoperative chemoradiotherapy with cisplatin and 5-FU improves the survival of patients with locally advanced adenocarcinoma of the cervix, with manageable toxicities. ${ }^{19}$ However, little has been reported on the effect of non-adherence to scheduled

Table 3 Acute toxicities stratified by patient group

\begin{tabular}{llll}
\hline & $\begin{array}{l}\text { Group I } \\
(\mathbf{n}, \%)\end{array}$ & $\begin{array}{l}\text { Group II } \\
(\mathbf{n}, \%)\end{array}$ & P-value \\
\hline Anemia & & & \\
$\quad$ Grade I-2 & $48,53.9$ & $44,65.7$ & 0.140 \\
$\quad$ Grade 3-4 & $3,3.4$ & $8,11.9$ & 0.038 \\
Neutropenia & & & \\
$\quad$ Grade I-2 & $74,83.1$ & $50,74.6$ & 0.192 \\
$\quad$ Grade 3-4 & $6,6.7$ & $14,20.9$ & 0.009 \\
$\begin{array}{l}\text { Thrombocytopenia } \\
\text { Grade I-2 }\end{array}$ & $7,7.9$ & $8,11.9$ & 0.393 \\
\hline
\end{tabular}

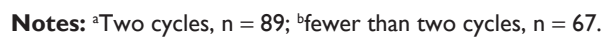

treatment of chemoradiotherapy with cisplatin and 5-FU every 3 weeks in locally advanced cervical cancer.

In the present study, only $57 \%$ of patients completed the planned protocol of two cycles of cisplatin and 5-FU, and we found that in terms of local control with a median 37.5-month follow-up less than two cycles was not as effective $(92.1 \%$ for those completing the regimen, compared to $80.6 \%$ of those who did not; $P=0.033$ ). However there were no statistically significant differences in disease free survival or overall survival between the two groups. Patients at stage IIB who did not complete the two planned cycles were also able to achieve 3-year local control, disease free survival, and metastasis free survival that was comparable to those who completed the regimen.

Table 4 Toxicities stratified by patient group (n, \%)

\begin{tabular}{|c|c|c|c|}
\hline & $\begin{array}{l}\text { Group Ia } \\
n\end{array}$ & $\begin{array}{l}\text { Group II } \\
n\end{array}$ & $P$-value \\
\hline \multicolumn{4}{|c|}{ Subcutaneous tissue } \\
\hline Grade I-2 & $23,25.8$ & $18,26.9$ & 0.886 \\
\hline Grade $3-4$ & 0,0 & $8,11.9$ & 0.001 \\
\hline \multicolumn{4}{|c|}{ Gastrointestinal } \\
\hline Grade I-2 & $7,7.9$ & $4,6.0$ & 0.647 \\
\hline Grade 3-4 & 0,0 & $2,3.0$ & 0.101 \\
\hline \multicolumn{4}{|l|}{ Genitourinary } \\
\hline Grade I-2 & $7,7.9$ & $5,7.5$ & 0.926 \\
\hline Grade 3-4 & 0,0 & I, I.5 & 0.248 \\
\hline
\end{tabular}

Notes: aTwo cycles, $n=89$; bfewer than two cycles, $n=67$. 
For patients in stage IIIB, there was also no statistical difference in 3-year local control, disease free survival, or overall survival between those who adhered to the regimen and those who could not.

We also found that compliance with treatment and acute hematologic toxicity were similar with the single chemotherapy agents with weekly cisplatin. ${ }^{6,12-14}$ In contrast, Kim et $\mathrm{al}^{20}$ reported that, compared to a monthly cisplatin plus 5-FU regimen, patients adhered more successfully to weekly cisplatin, which reduced acute hematologic toxicity and had no effect on response and survival rates. According to the results of several studies, treatment outcomes for cisplatin alone and the cisplatin plus 5-FU regimen are similar, but there are considerable differences in acute toxicity and compliance. ${ }^{14}$ We found no occurrence of nephrotoxicity in the present study. However, in a study of 165 patients treated with cisplatin and chemoradiation, Tharavichitkul et $\mathrm{al}^{6}$ reported nephrotoxicity in 14 patients, and three patients developed grade 3-4 toxicity.

In our study, completion of two cycles of 5-FU/cisplatin and radiotherapy significantly improved local control, when considering the entire patient group. Those patients who did not complete treatment had significantly more anemia and neutropenia, and these side effects were, in general, the cause of non-compliance. Further investigation of patients' inability to complete scheduled chemoradiation revealed that adverse side effects, patient motivation, and laboratory profiles all contributed to lack of success. We recommend that for locally advanced cervical cancer, clinicians consider cytokine and blood product support to improve patient compliance with chemotherapy, and that total treatment time should be kept to the minimum required to optimize therapy. These findings are in accord with the literature as well.

Our results are limited by the study's retrospective design and because data was obtained from a single institution. The sample size is probably insufficient for subgroup calculations, although the completed chemoradiotherapy regimen showed a marked advantage in local control. The sample size of the study was determined by local patient volume, and we cannot exclude the possibility that other factors such as tumor size might be significant correlates of survival. We believe that by adjusting treatment for tumor size, we compensated to some extent for its adverse prognostic import. Yet the findings of this study confirm our clinical impressions and provide important information with which to move forward in developing better therapies for locally advanced cervical cancer.

\section{Conclusion}

In conclusion, we found that chemoradiotherapy with 5-FU and cisplatin was associated with tolerable acute and longterm toxicity, and is safe and effective. In women with locally advanced cervical cancer, completed chemoradiotherapy with two cycles of cisplatin and 5-FU improved local control over incomplete treatment. No differences were found in disease free and overall survival. The present study supports the significant need for patients to adhere to chemoradiation therapy.

\section{Acknowledgments}

This work is supported by the Foundation of the First Affiliated Hospital of Medical College of Xi'an Jiaotong University (NO 2010YK3) and the National Natural Science Foundation of China (NO 81071838).

\section{Disclosure}

The authors report no conflicts of interest in this work.

\section{References}

1. National Comprehensive Cancer Center (NCCN). NCCN Clinical Practice Guidelines in Oncology: Cervical Cancer, Version 2. 2013. Fort Washington, PA: NCCN; 2012. Available from: https://subscriptions. nccn.org/gl_login.aspx?ReturnURL=http://www.nccn.org/professionals/ physician_gls/pdf/cervical.pdf. Accessed January 30, 2013.

2. Laurentius T, Altendorf-Hofmann A, Camara O, Runnebaum IB, Wendt TG. Impact of age on morbidity and outcome of concurrent radiochemotherapy in high-risk FIGO stage I to IVA carcinoma of the uterine cervix following laparoscopic surgery. J Cancer Res Clin Oncol. 2011;137(3):481-488.

3. Ikushima H, Osaki K, Furutani S, et al. Chemoradiation therapy for cervical cancer: toxicity of concurrent weekly cisplatin. Radiat Med. 2006;24(2):115-121.

4. Nugent EK, Case AS, Hoff JT, et al. Chemoradiation in locally advanced cervical carcinoma: an analysis of cisplatin dosing and other clinical prognostic factors. Gynecol Oncol. 2010;116(3):438-441.

5. Geara FB, Shamseddine A, Khalil A, Abboud M, Charafeddine M, Seoud M. A phase II randomized trial comparing radiotherapy with concurrent weekly cisplatin or weekly paclitaxel in patients with advanced cervical cancer. Radiat Oncol. 2010;5:84.

6. Tharavichitkul E, Pinitpatcharalerd A, Lorvidhaya V, et al. Impact of incomplete plan to treatment results of concurrent weekly cisplatin and radiotherapy in locally advanced cervical cancer. $J$ Radiat Res. 2011;52(1):9-14.

7. National Cancer Institute (March 31, 2003). Cancer Therapy Evaluation Program. Common Terminology Criteria for Adverse Events. Version 3.0. DCTD, NCI, NIH, NHHH. Available from http://cteo.cancer.gov.

8. Keys HM, Bundy BN, Stehman FB, et al. Cisplatin, radiation, and adjuvant hysterectomy compared with radiation and adjuvant hysterectomy for bulky stage IB cervical carcinoma. $N$ Engl $J$ Med. 1999;340(15):1154-1161.

9. Morris M, Eifel PJ, Lu J, et al. Pelvic radiation with concurrent chemotherapy compared with pelvic and para-aortic radiation for high-risk cervical cancer. $N$ Engl J Med. 199;340(15):1137-1143.

10. Peters WA 3rd, Liu PY, Barrett RJ 2nd, et al. Concurrent chemotherapy and pelvic radiation therapy compared with pelvic radiation therapy alone as adjuvant therapy after radical surgery in high-risk early-stage cancer of the cervix. J Clin Oncol. 2000;18(8):1606-1613. 
11. Rose PG, Bundy BN, Watkins EB, et al. Concurrent cisplatin-based radiotherapy and chemotherapy for locally advanced cervical cancer. N Engl J Med. 1999;340(15):1144-1153.

12. Whitney CW, Sause W, Bundy BN, et al. Randomized comparison of fluorouracil plus cisplatin versus hydroxyurea adjunct to radiation therapy in stage IIB-IVA carcinoma of the cervix with negative para-aortic lymph nodes: a Gynecologic Oncology Group and South west Oncology Group study. J Clin Oncol. 1999;17(5):1339-1348.

13. Monk BJ, Tian C, Rose PG, Lanciano R. Which clinical/pathologic factors matter in the era of chemoradiation as treatment for locally advanced cervical carcinoma? Analysis of two Gynecologic Oncology Group (GOG) trials. Gynecol Oncol. 2007;105(2):427-433.

14. Lanciano R, Calkins A, Bundy BN, et al. Randomized comparison of weekly cisplatin or protracted venous infusion of fluorouacil in combination with pelvic radiation in advanced cervix cancer: a gynecologic oncology group study. J Clin Oncol. 2005;23(33):8289-8295.

15. Ryu SY, Lee WM, Kim K, et al. Randomized clinical trial of weekly vs triweekly cisplatin-based chemotherapy concurrent with radiotherapy in the treatment of locally advanced cervical cancer. Int J Radiat Oncol Biol Phys. 2011;81(4):e577-e581.
16. Morris M, Eifel PJ, Lu J, et al. Pelvic radiation with concurrent chemotherapy compared with pelvic and para-aortic radiation for highrisk cervical cancer. $N$ Engl J Med. 1999;340(15):1137-1143.

17. Ooyama A, Oka T, Zhao HY, Yamamoto M, Akiyama S, Fukushima M. Anti-angiogenic effect of 5-Fluorouracil-based drugs against human colon cancer xenografts. Cancer Lett. 2008; 267(1):26-36.

18. Nagai N, Mukai K, Hirata E, Jin HH, Komatsu M, Yunokawa M. UFT and its metabolite gamma-butyrolactone (GBL) inhibit angiogenesis induced by vascular endothelial growth factor in advanced cervical carcinoma. Med Oncol. 2008;25(2):214-221.

19. Shibata K, Kajiyama H, Yamamoto E, et al. Effectiveness of preoperative concurrent chemoradiation therapy (CCRT) for locally advanced adenocarcinoma of cervix. Eur J Surg Oncol. 2009;35(7):768-772.

20. Kim YS, Shin SS, Nam JH, et al. Prospective randomized comparison of monthly fluorouracil and cisplatin versus weekly cisplatin concurrent with pelvic radiotherapy and high-dose rate brachytherapy for locally advanced cervical cancer. Gynecol Oncol. 2008;108(1):195-200.
OncoTargets and Therapy

\section{Publish your work in this journal}

OncoTargets and Therapy is an international, peer-reviewed, open access journal focusing on the pathological basis of all cancers, potential targets for therapy and treatment protocols employed to improve the management of cancer patients. The journal also focuses on the impact of management programs and new therapeutic agents and protocols on

\section{Dovepress}

patient perspectives such as quality of life, adherence and satisfaction The manuscript management system is completely online and includes a very quick and fair peer-review system, which is all easy to use. Visit http://www.dovepress.com/testimonials.php to read real quotes from published authors. 